\title{
Three-dimensional quasistatic model for high brightness beam dynamics simulation
}

\author{
Ji Qiang, Steve Lidia, and Robert D. Ryne \\ Lawrence Berkeley National Laboratory, Berkeley, California 94720, USA \\ Cecile Limborg-Deprey \\ Stanford Linear Accelerator Center, Menlo Park, California 94309, USA
}

(Received 9 November 2005; published 27 April 2006)

\begin{abstract}
In this paper, we present a three-dimensional quasistatic model for high brightness beam dynamics simulation in $\mathrm{rf} / \mathrm{dc}$ photoinjectors, $\mathrm{rf}$ linacs, and similar devices on parallel computers. In this model, electrostatic space-charge forces within a charged particle beam are calculated self-consistently at each time step by solving the three-dimensional Poisson equation in the beam frame and then transforming back to the laboratory frame. When the beam has a large energy spread, it is divided into a number of energy bins or slices so that the space-charge forces are calculated from the contribution of each bin and summed together. Image-charge effects from conducting photocathode are also included efficiently using a shifted-Green function method. For a beam with large aspect ratio, e.g., during emission, an integrated Green function method is used to solve the three-dimensional Poisson equation. Using this model, we studied beam transport in one Linac Coherent Light Sources photoinjector design through the first traveling wave linac with initial misalignment with respect to the accelerating axis.
\end{abstract}

DOI: 10.1103/PhysRevSTAB.9.044204

PACS numbers: 29.27.Bd

\section{INTRODUCTION}

High brightness, low emittance electron beams produced from photoinjectors have important applications in next generation light sources and linear colliders. Simplified theoretical models have been used to provide some guidelines for photoinjector design [1-3]. However, systematic machine design and optimization still depend heavily on the use of self-consistent computer simulations. A number of computer models have been used in the past years to simulate beam transport inside photoinjectors [412]. As far as we know, a model which can efficiently and accurately handle a three-dimensional beam with large aspect ratio and large energy spread including possible image-charge effects has not previously been available on parallel computers. Although most photoinjector designs assume azimuthal symmetry of the beam and the $\mathrm{rf}$ accelerating structures, experimental measurements at the exit of the injector have shown asymmetric structure within the beam [13-15]. This could be due to inhomogeneous emission of electrons from the photocathode. It could also be due to misalignment between the laser spot on the photocathode and the rf accelerating structure axis. The asymmetric rf or solenoid field errors inside photoinjector could also break the azimuthal symmetry of the beam and present a fully three-dimensional problem. An accurate three-dimensional model will help one to understand the beam transport inside the photoinjector and to study the error tolerance for these photoinjectors. In the process of electron emission from the photocathode, the transverse beam size is typically on the order of millimeters, whereas the typical longitudinal beam size can be about tens to hundreds of microns. At this stage, the beam has a very large transverse-to-longitudinal aspect ratio. After the beam has been accelerated to a few $\mathrm{MeV}$, the longitudinal beam size is on the same order as the transverse beam size. This results in a large longitudinal-to-transverse aspect ratio in the beam frame due to the relativistic effects. In both cases, it requires accurate modeling of a threedimensional beam with large-aspect ratio. Meanwhile, the demand of highly asymmetric beam for next generation linear colliders will also require accurately modeling a three-dimensional beam with large aspect ratio. In this paper, we report on developing a three-dimensional quasistatic particle-in-cell model to simulate the high brightness beam transport through a rf structure (e.g., photoinjector) using a shifted integrated Green function method for spacecharge calculation [16]. Using a shifted integrated Green function, we can accurately and efficiently calculate the three-dimensional space-charge forces of a beam with large aspect ratio and the image-charge effects with arbitrary separation using a fast Fourier transform (FFT) method. In some applications with long laser pulse and high rf accelerating gradient, the electrons out of the photocathode could have a large correlated energy spread. This large energy spread in the laboratory frame is handled by dividing the beam into multiple energy bins/slices and the space-charge forces are calculated for each slice and are summed together before being interpolated to individual particles. Besides the efficient numerical algorithm for calculation of the 3D space-charge forces, we have also implemented this model on parallel computers using a domain-decomposition method. This enables us to run a simulation with both high resolution (e.g., a large number of macroparticles) and fast return time. 


\section{PHYSICAL MODEL AND COMPUTATIONAL METHODS}

The physical model assumed here is a relativistic quasistatic charged particle beam subject to external acceleration and focusing and space-charge forces from the beam itself (here, the quasistatic means that the static electric field is calculated in the beam frame and both electric and transverse magnetic fields are included in the laboratory frame). Radiation effects of the beam are not included. Comparison studies with and without the radiation effects suggest that the radiation effects should not be significant for the current generation of photoinjectors $[17,18]$. The simulation starts by generating a three-dimensional beam bunch behind the photocathode according to the laser pulse length and distribution. This bunch is then moved out of the cathode within the given emission time. During the process of emission, the space-charge forces of particles outside the cathode are included together with external fields from the rf cavity and solenoid magnet. Besides the rf/dc cavity and solenoid magnet beam line elements, the model also includes dipole, quadrupole, sextupole, octupole, and decapole magnets in the beam line element list. External fields can be supplied in the form of discrete data on a three-dimensional Cartesian coordinate mesh or a cylindrical coordinate mesh. They can also be supplied in the form of Fourier expansion coefficients on the axis when the fields have azimuthal symmetry. These elements can be arranged with arbitrary longitudinal overlap so that a traveling wave structure (including coupling cells) can be modeled using two overlapped standing wave structures and an input and an output standing wave structures [19].

The particles inside a photoinjector are advanced selfconsistently using a particle-in-cell approach. The general equations of motion are

$$
\begin{gathered}
\dot{\mathbf{r}}=\frac{\mathbf{p}}{m \gamma}, \\
\dot{\mathbf{p}}=q\left(\mathbf{E}+\frac{\mathbf{p}}{m \gamma} \times \mathbf{B}\right),
\end{gathered}
$$

where, $\mathbf{r}$ is spatial position vector, $\mathbf{p}$ is mechanic momentum vector, $\gamma=1 / \sqrt{1-\beta^{2}}, \beta^{2}=\sum \beta_{i}^{2}, \beta_{i}=v_{i} / c$ with $i=x, y, z, c$ is the speed of light, $m$ is the rest mass of particle, $q$ is the charge of particle. The electric field, $\mathbf{E}$, and magnetic field, $\mathbf{B}$, include the contributions from external focusing and accelerating fields and space-charge fields of intraparticle Coulomb interactions. For an rf linac, the external electromagnetic fields in a cylindrically symmetric accelerating structure can be obtained from the field distribution along the axis [20,21]. If the rf cavity does not have a cylindrical symmetry, the fully three-dimensional electromagnetic fields are read in from the external supplied data files. The equations of motion are solved using a second-order leap-frog algorithm: the particles are drifted half time step; the particles are collected and deposited onto a three-dimensional grid; the Poisson equation is solved in the beam frame; the electric and magnetic fields are obtained in the laboratory frame through the Lorentz transformation; the particle momenta are updated using both the space-charge fields and external fields for one time step according to Eq. (2); the particles are drifted another half time step. This procedure is repeated for many time steps until the beam is out of the computational domain.

To calculate the space-charge forces, we solve the threedimensional Poisson equation. The solution of Poisson's equation can be written as

$$
\begin{aligned}
\phi(x, y, z)= & \frac{1}{4 \pi \epsilon_{0}} \\
& \times \int G\left(x, x^{\prime}, y, y^{\prime}, z, z^{\prime}\right) \rho\left(x^{\prime}, y^{\prime}, z^{\prime}\right) d x^{\prime} d y^{\prime} d z^{\prime},
\end{aligned}
$$

where $G$ is Green's function, $\rho$ is the charge density distribution function. For a high brightness beam inside a photoinjector, the conducting pipe is normally much larger than the size of the beam. In this case, an open boundary condition except near the conducting photocathode can be assumed for the solution of the Green's function in above equation. Here, the Green function is given by

$$
G\left(x, x^{\prime}, y, y^{\prime}, z, z^{\prime}\right)=\frac{1}{\sqrt{\left(x-x^{\prime}\right)^{2}+\left(y-y^{\prime}\right)^{2}+\left(z-z^{\prime}\right)^{2}}} .
$$

Now consider a simulation of an open system where the computational domain containing the particles has a range of $\left(0, L_{x}\right),\left(0, L_{y}\right)$, and $\left(0, L_{z}\right)$, and where each dimension has been discretized using $N_{x}, N_{y}$, and $N_{z}$ points. From Eq. (3), the electric potentials on the grid can be approximated as

$$
\phi\left(x_{i}, y_{j}, z_{k}\right)=\frac{h_{x} h_{y} h_{z}}{4 \pi \epsilon_{0}} \sum_{i^{\prime}=1}^{N_{x}} \sum_{j^{\prime}=1}^{N_{y}} \sum_{k^{\prime}=1}^{N_{z}} G\left(x_{i}-x_{i^{\prime}}, y_{j}-y_{j^{\prime}}, z_{k}-z_{k^{\prime}}\right) \rho\left(x_{i^{\prime}}, y_{j^{\prime}}, z_{k^{\prime}}\right),
$$

where $x_{i}=(i-1) h_{x}, y_{j}=(j-1) h_{y}$, and $z_{k}=(k-1) h_{z}$. As discussed later, a more accurate approximation to Eq. (3) is needed for a beam with large aspect ratio. This is possible by using an integrated Green function method. The computational cost of the above convolution by a direct numerical summation can be very expensive and scales as $N^{6}$, where $N$ is the number of grid points in each dimension. Fortunately, this convolution can be replaced by a cyclic convolution in a 
double-gridded computational domain:

$$
\phi_{c}\left(x_{i}, y_{j}, z_{k}\right)=\frac{h_{x} h_{y} h_{z}}{4 \pi \epsilon_{0}} \sum_{i^{\prime}=1}^{2 N_{x}} \sum_{j^{\prime}=1}^{2 N_{y}} \sum_{k^{\prime}=1}^{2 N_{z}} G_{c}\left(x_{i}-x_{i^{\prime}}, y_{j}-y_{j^{\prime}}, z_{k}-z_{k^{\prime}}\right) \rho_{c}\left(x_{i^{\prime}}, y_{j^{\prime}}, z_{k^{\prime}}\right),
$$

where $i=1, \ldots, 2 N_{x}, j=1, \ldots, 2 N_{y}, k=1, \ldots, 2 N_{z}$ and

$$
\begin{gathered}
\rho_{c}\left(x_{i}, y_{j}, z_{k}\right)= \begin{cases}\rho\left(x_{i}, y_{j}, z_{k}\right): & 1 \leq i \leq N_{x} ; 1 \leq j \leq N_{y} ; 1 \leq k \leq N_{z}, \\
0: & N_{x}<i \leq 2 N_{x} \text { or } N_{y}<j \leq 2 N_{y} \text { or } N_{z}<k \leq 2 N_{z},\end{cases} \\
G_{c}\left(x_{i}, y_{j}, z_{k}\right)= \begin{cases}G\left(x_{i}, y_{j}, z_{k}\right): & 1 \leq i \leq N_{x}+1 ; 1 \leq j \leq N_{y}+1 ; 1 \leq k \leq N_{z}+1, \\
G\left(x_{2 N_{x}-i+2}, y_{j}, z_{k}\right): & N_{x}+1<i \leq 2 N_{x} ; 1 \leq j \leq N_{y}+1 ; 1 \leq k \leq N_{z}+1, \\
G\left(x_{i}, y_{2 N_{y}-j+2}, z_{k}\right): & 1 \leq i \leq N_{x}+1 ; N_{y}+1<j \leq 2 N_{y} ; 1 \leq k \leq N_{z}+1, \\
G\left(x_{2 N_{x}-i+2}, y_{2 N_{y}-j+2}, z_{k}\right): & N_{x}+1<i \leq 2 N_{x} ; N_{y}+1<j \leq 2 N_{y} ; 1 \leq k \leq N_{z}+1, \\
G\left(x_{i}, y_{j}, z_{\left.2 N_{z}-k+2\right)}\right) & 1 \leq i \leq N_{x}+1 ; 1 \leq j \leq N_{y}+1 ; N_{z}+1<k \leq 2 N_{z}, \\
G\left(x_{2 N_{x}-i+2}, y_{j}, z_{2 N_{z}-k+2}\right): & N_{x}+1<i \leq 2 N_{x} ; 1 \leq j \leq N_{y}+1 ; N_{z}+1<k \leq 2 N_{z}, \\
G\left(x_{i}, y_{2 N_{y}-j+2}, z_{2 N_{z}-k+2}\right): & 1 \leq i \leq N_{x}+1 ; N_{y}+1<j \leq 2 N_{y} ; N_{z}+1<k \leq 2 N_{z}, \\
G\left(x_{2 N_{x}-i+2}, y_{2 N_{y}-j+2}, z_{2 N_{z}-k+2}\right): & N_{x}+1<i \leq 2 N_{x} ; N_{y}+1<j \leq 2 N_{y} ; N_{z}+1<k \leq 2 N_{z},\end{cases} \\
\quad \rho_{c}\left(x_{i}, y_{j}, z_{k}\right)=\rho_{c}\left[x_{i}+2\left(L_{x}+h_{x}\right), y_{j}+2\left(L_{y}+h_{y}\right), z_{k}+2\left(L_{z}+h_{z}\right)\right], \\
G_{c}\left(x_{i}, y_{j}, z_{k}\right)=G_{c}\left[x_{i}+2\left(L_{x}+h_{x}\right), y_{j}+2\left(L_{y}+h_{y}\right), z_{k}+2\left(L_{z}+h_{z}\right)\right] .
\end{gathered}
$$

These equations make use of the symmetry of the Green function in Eq. (4). From the above definition, one can show that the cyclic convolution will give the same electric potential as the convolution Eq. (5) within the original domain, i.e.,

$$
\begin{aligned}
& \phi\left(x_{i}, y_{j}, z_{k}\right)=\phi_{c}\left(x_{i}, y_{j}, z_{k}\right) \\
& \quad \text { for } i=1, N_{x} ; j=1, N_{y} ; k=1, N_{z} .
\end{aligned}
$$

The potential outside the original domain is incorrect but is irrelevant to the physical domain. Since now both $G_{c}$ and $\rho_{c}$ are periodic functions, the convolution for $\phi_{c}$ in Eq. (6) can be computed efficiently using an FFT as described by Hockney and Eastwood [22]. The computational cost of this algorithm scales as $N^{4} \log (N)$ instead of the $N^{6}$ in the direct summation.

For a conducting photocathode, the space-charge forces both from the image charge and from the beam itself are included. To find the forces on the beam from the image charge by the standard Green function method, we need to solve the Poisson equation with a computational domain containing both the image charge and the beam. This can be very inefficient since only the fields within the beam are needed and the fields outside of the beam are not used. This inefficiency can be avoided by using a shifted-Green function method [16]. The shifted-Green function method is based on an observation that the potential $\phi(X, Y, Z)$ inside the field domain, where $x_{c}-x_{\text {left }} \leq X \leq x_{c}+x_{\text {right }}, y_{c}-$ $y_{\text {left }} \leq Y \leq y_{c}+y_{\text {right }}, z_{c}-z_{\text {left }} \leq Z \leq z_{c}+z_{\text {right }}$, can be rewritten as $\phi\left(x+x_{c}, y+y_{c}, z+z_{c}\right)$, where $-x_{\text {left }} \leq x \leq$ $x_{\text {right }},-y_{\text {left }} \leq y \leq+y_{\text {right }},-z_{\text {left }} \leq z \leq z_{\text {right }}$. Here, $x_{c}$, $y_{c}$, and $z_{c}$ are coordinates of the field domain center with respect to the coordinate origin in the particle domain, $x_{\text {left }}$, $x_{\text {right }}, y_{\text {left }}, y_{\text {right }}, z_{\text {left }}$, and $z_{\text {right }}$ are sizes of the field domain measured with respect to its center in each dimension. These sizes must also be large enough to cover the particle domain which contains the image charged particles. In these new coordinates, the original Green function in Eq. (4) can be written as a shifted-Green function

$$
G_{s}\left(x, x^{\prime}, y, y^{\prime}, z, z^{\prime}\right)=\frac{1}{\sqrt{\left(x_{c}+x-x^{\prime}\right)^{2}+\left(y_{c}+y-y^{\prime}\right)^{2}+\left(z_{c}+z-z^{\prime}\right)^{2}}} .
$$

The electric potential in the field domain is written as

$$
\phi\left(x+x_{c}, y+y_{c}, z+z_{c}\right)=\frac{1}{4 \pi \epsilon_{0}} \int G_{s}\left(x, x^{\prime}, y, y^{\prime}, z, z^{\prime}\right) \rho\left(x^{\prime}, y^{\prime}, z^{\prime}\right) d x^{\prime} d y^{\prime} d z^{\prime},
$$

where $\rho$ is the image-charge density distribution. This convolution can be computed following the same procedure as the standard Green function method except the use of a shifted-Green function. The new cyclic Green function for the shiftedGreen function on the doubled computational grid is given as 


$$
G_{s c}\left(x_{i}, y_{j}, z_{k}\right)= \begin{cases}{\left[\left(x_{c}+x_{i}\right)^{2}+\left(y_{c}+y_{j}\right)^{2}+\left(z_{c}+z_{k}\right)^{2}\right]^{-1 / 2}:} & 1 \leq i \leq N_{x} ; 1 \leq j \leq N_{y} ; 1 \leq k \leq N_{z}, \\ {\left[\left(x_{c}-x_{2 N_{x}-i+2}\right)^{2}+\left(y_{c}+y_{j}\right)^{2}+\left(z_{c}+z_{k}\right)^{2}\right]^{-1 / 2}:} & N_{x}<i \leq 2 N_{x} ; 1 \leq j \leq N_{y} ; 1 \leq k \leq N_{z}, \\ {\left[\left(x_{c}+x_{i}\right)^{2}+\left(y_{c}-y_{2 N_{y}-j+2}\right)^{2}+\left(z_{c}+z_{k}\right)^{2}\right]^{-1 / 2}:} & 1 \leq i \leq N_{x} ; N_{y}<j \leq 2 N_{y} ; 1 \leq k \leq N_{z}, \\ {\left[\left(x_{c}-x_{2 N_{x}-i+2}\right)^{2}+\left(y_{c}-y_{2 N_{y}-j+2}\right)^{2}+\left(z_{c}+z_{k}\right)^{2}\right]^{-1 / 2}:} & N_{x}<i \leq 2 N_{x} ; N_{y}<j \leq 2 N_{y} ; 1 \leq k \leq N_{z}, \\ {\left[\left(x_{c}+x_{i}\right)^{2}+\left(y_{c}+y_{j}\right)^{2}+\left(z_{c}-z_{2 N_{z}-k+2}\right)^{2}\right]^{-1 / 2}:} & 1 \leq i \leq N_{x} ; 1 \leq j \leq N_{y} ; N_{z}<k \leq 2 N_{z}, \\ {\left[\left(x_{c}-x_{2 N_{x}-i+2}\right)^{2}+\left(y_{c}+y_{j}\right)^{2}+\left(z_{c}-z_{2 N_{z}-k+2}\right)^{2}\right]^{-1 / 2}:} & N_{x}<i \leq 2 N_{x} ; 1 \leq j \leq N_{y} ; N_{z}<k \leq 2 N_{z}, \\ {\left[\left(x_{c}+x_{i}\right)^{2}+\left(y_{c}-y_{2 N_{y}-j+2}\right)^{2}+\left(z_{c}-z_{2 N_{z}-k+2}\right)^{2}\right]^{-1 / 2}:} & 1 \leq i \leq N_{x} ; N_{y}<j \leq 2 N_{y} ; N_{z}<k \leq 2 N_{z}, \\ {\left[\left(x_{c}-x_{2 N_{x}-i+2}\right)^{2}+\left(y_{c}-y_{2 N_{y}-j+2}\right)^{2}+\left(z_{c}-z_{2 N_{z}-k+2}\right)^{2}\right]^{-1 / 2}:} & N_{x}<i \leq 2 N_{x} ; N_{y}<j \leq 2 N_{y} ; N_{z}<k \leq 2 N_{z} .\end{cases}
$$

The FFT used to calculate the cyclic convolution in Hockney's algorithm for standard Green function can be used to calculate the potential in the field domain using the new shifted-Green function. This avoids the requirement that the particle domain and the field domain be contained in one large computational domain. This leads to improved numerical resolution for the charge densities and the resulting electric fields than the conventional method, because the empty space between the charged bunches is not included in the calculation. It is also far more efficient, in terms of computational effort and storage, than the traditional approach of gridding the entire problem domain. To test this algorithm, we have calculated the potential distribution on the axis from the image charge of a round beam as shown in Fig. 1. Here, the $1 \mathrm{nC}$ electron charge has a 3D waterbag distribution with $1 \mathrm{~mm}$ rms size ( $\sqrt{8} \mathrm{~mm}$ radius) and located $5 \mathrm{~mm}$ after the cathode. The numerical solution of the electrical potential using the shifted-Green function method is given in Fig. 2 together with the analytical solution. It is seen that the numerical solution and the analytical solution agree with each other very well.

The image charge of a beam can have significant effects on the beam quality in photoinjectors. Using the Linac Coherent Light Sources (LCLS) $S$-band rf gun [23], we did simulations with and without the image space-charge effects. Here, the initial distribution of electron bunch is a cold 10 ps long uniform cylinder with $1 \mathrm{~mm}$ radius. The

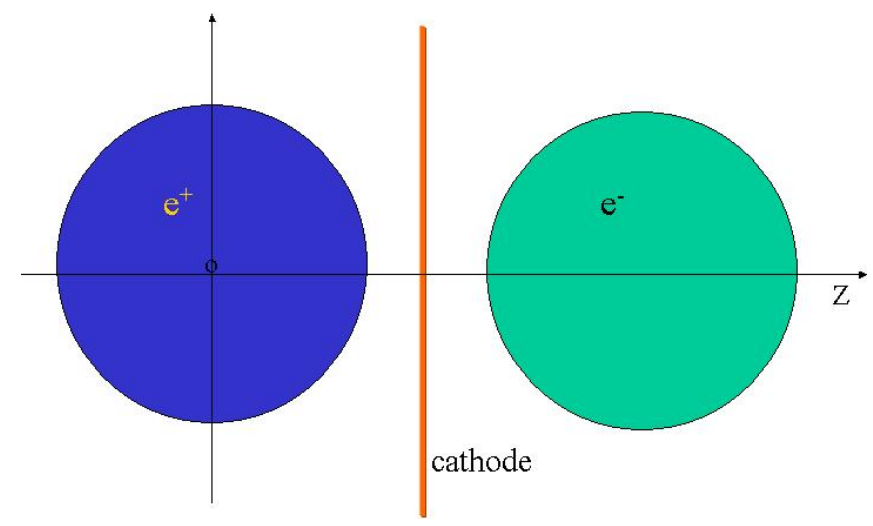

FIG. 1. (Color) A schematic plot of an electron bunch in front of the conducting photocathode together with its image charge. total charge in this bunch is $1 \mathrm{nC}$. The peak acceleration field is $120 \mathrm{MV} / \mathrm{m}$. Figure 3 shows the transverse and longitudinal rms sizes of the bunch with and without the image-charge effects of the conducting photocathode in the simulation. It is seen that the beam without image charge has a larger initial transverse size than the beam with image charge. The space-charge forces from the image charge of the beam have opposite directions compared with the space-charge forces from the beam itself. This helps to reduce the initial beam blowup driven by the space-charge forces.

In the above algorithms, both the Green function and the charge density distribution are discretized on the grid. For a beam with an aspect ratio close to 1 , this algorithm works well. However, during the emission of electrons out of the cathode, the beam can have a very large transverse-tolongitudinal ratio. For example, the typical transverse size is on the order of millimeters while the longitudinal size can be about a few tens to hundreds of microns. Under this situation, the direct use of the Green function at each mesh point is not efficient since it requires a large number of mesh points along the transverse direction in order to get sufficient resolution for the Green function along that direction. A two-dimensional integrated Green function

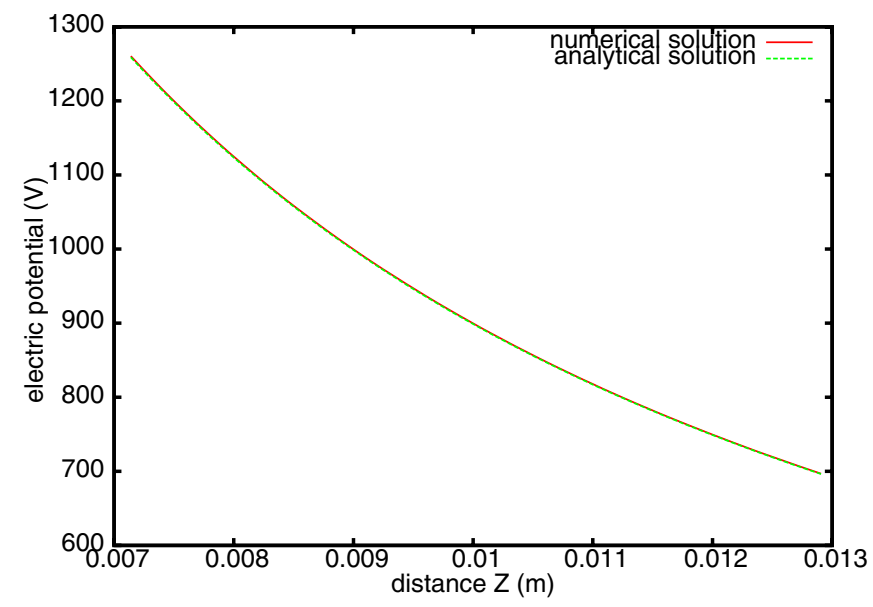

FIG. 2. (Color) Image-charge electric potential on the axis for a round beam from the shifted-Green function solution together with the analytical solution. 

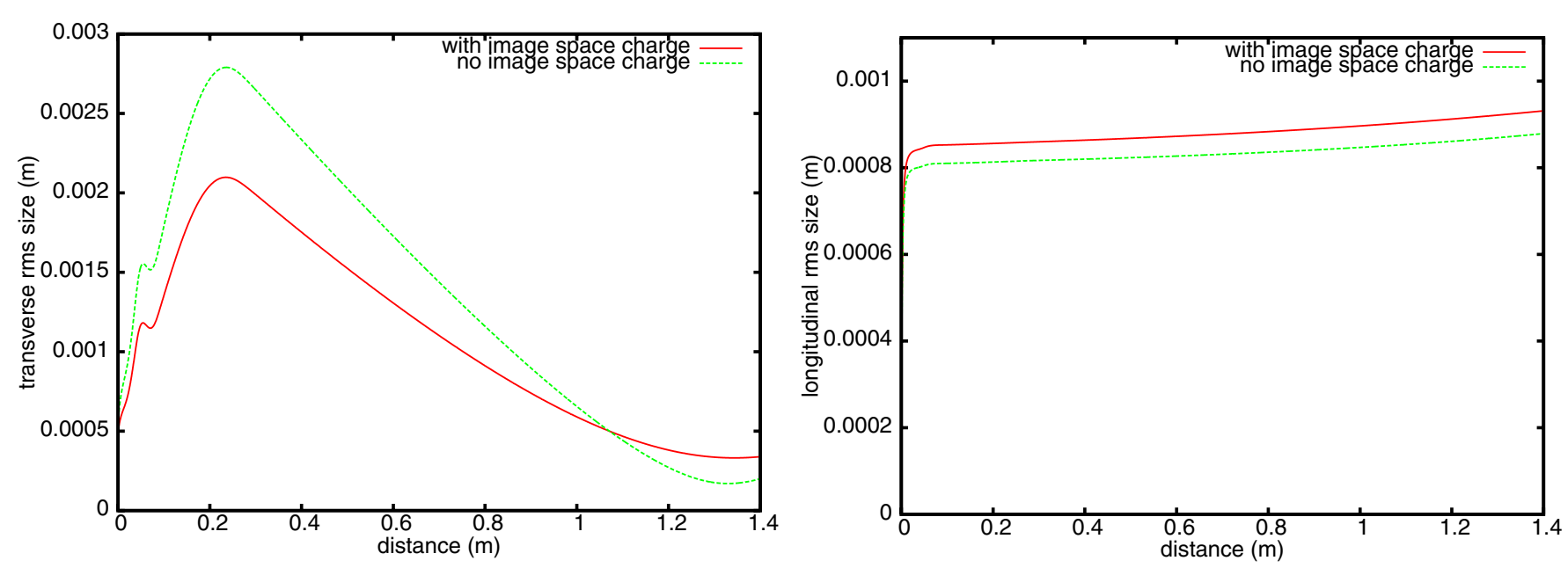

FIG. 3. (Color) Transverse (left) and longitudinal (right) rms sizes as a function of distance with and without the image-charge effects from photocathode.

method has successfully been used in previous studies of the beam with large aspect ratio [16,24-26]. Here, we generalize that method to three-dimensional space-charge force calculations. If we assume that the charge density function is uniform within each cell centered at the grid point $\left(x_{i}, y_{j}, z_{k}\right)$, we can define an effective Green function as

$$
\left.\bar{G}_{\left(x_{i}\right.}-x_{i^{\prime}}, y_{j}-y_{j^{\prime}}, z_{k}-z_{k^{\prime}}\right)=\int_{x_{i^{\prime}}-h_{x} / 2}^{x_{i^{\prime}}+h_{x} / 2} d x^{\prime} \int_{y_{j^{\prime}}-h_{y} / 2}^{y_{j^{\prime}}+h_{y} / 2} d y^{\prime} \int_{z_{k^{\prime}}-h_{z} / 2}^{z_{k^{\prime}}+h_{z} / 2} d z^{\prime} G\left(x_{i}-x^{\prime}, y_{j}-y^{\prime}, z_{k}-z^{\prime}\right) .
$$

This integral can be calculated analytically in a closed form:

$$
\begin{aligned}
\iiint \frac{1}{\sqrt{x^{2}+y^{2}+z^{2}} d x d y d z=} & y z \ln \left(x+\sqrt{x^{2}+y^{2}+z^{2}}\right)+x z \ln \left(y+\sqrt{x^{2}+y^{2}+z^{2}}\right)+x y \ln \left(z+\sqrt{x^{2}+y^{2}+z^{2}}\right) \\
& -\frac{1}{4} i z^{2} \ln \left(\frac{-8 i x^{2}+8(y-i z)-8 i x \sqrt{x^{2}+y^{2}+z^{2}}}{x^{2}(y-i z) z^{2}}\right) \\
& +\frac{1}{4} i z^{2} \ln \left(\frac{8 i x^{2}+8(y+i z)+8 i x \sqrt{x^{2}+y^{2}+z^{2}}}{x^{2}(y+i z) z^{2}}\right) \\
& +\frac{1}{4} i x^{2} \ln \left(\frac{8\left[x^{2}-i x y+z\left(z+\sqrt{x^{2}+y^{2}+z^{2}}\right)\right]}{x^{2}(x-i y) z^{2}}\right) \\
& -\frac{1}{4} i x^{2} \ln \left(\frac{8\left[x^{2}+i x y+z\left(z+\sqrt{x^{2}+y^{2}+z^{2}}\right)\right]}{x^{2}(x+i y) z^{2}}\right) \\
& -\frac{1}{4} i y^{2} \ln \left(\frac{2 x y-2 i\left[y^{2}+z\left(z+\sqrt{x^{2}+y^{2}+z^{2}}\right)\right]}{y(x-i y) z^{2}}\right) \\
& +\frac{1}{4} i y^{2} \ln \left(\frac{2 x y+2 i\left[y^{2}+z\left(z+\sqrt{x^{2}+y^{2}+z^{2}}\right)\right]}{y(x+i y) z^{2}}\right) .
\end{aligned}
$$

In the above algorithm, we assumed a uniform density distribution within each cell. It is also possible to assume a trilinear density distribution inside a cell. As a test of this algorithm, we calculated the electric fields along the $x$ axis and $z$ axis of a charged beam with uniform density distribution. The numerical results from the integrated Green function together with the solutions from the standard Green function method and the analytical method are given in Figs. 4 and 5 for two transverse-to-longitudinal aspect ratios. Here, $128 \times 128 \times 128$ computational grid points have been used. In Fig. 4, the aspect ratio is 1, which corresponds to a uniform spherical ball, all three solutions agree with each other very well. In Fig. 5, the aspect ratio is 30 , we can see that the integrated Green function method and the analytical method agree with each other very well. The standard Green function method gives significant dis- 

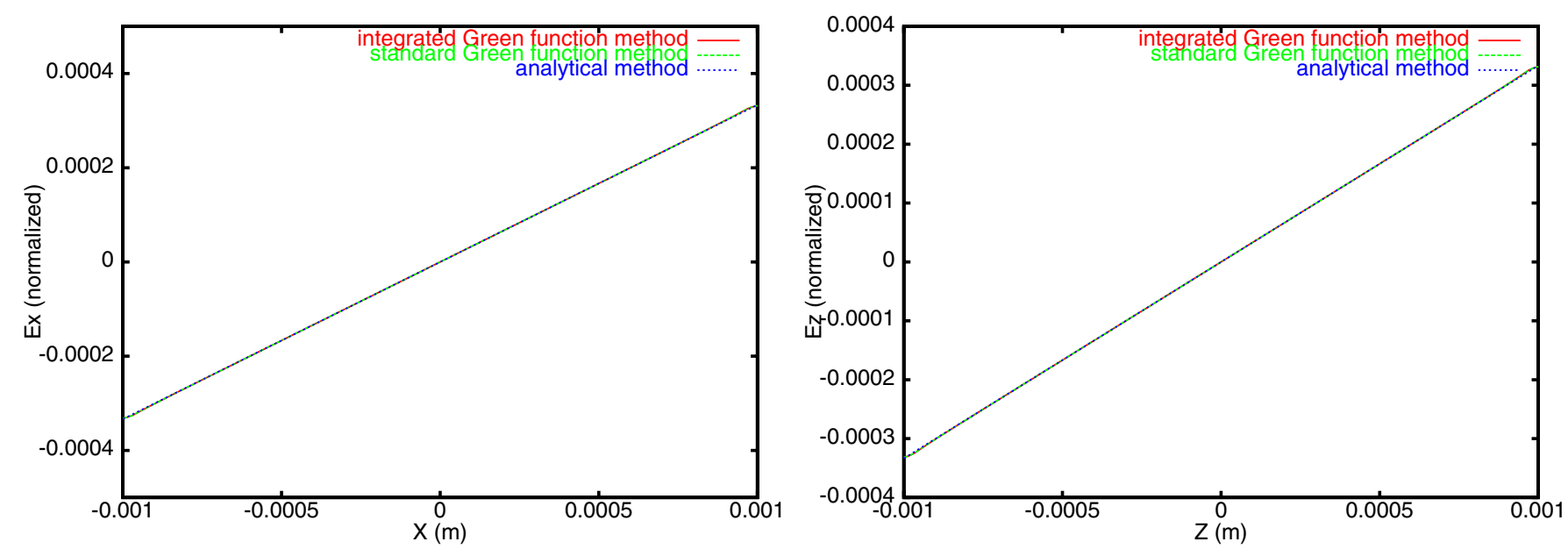

FIG. 4. (Color) Electric fields along $x$ axis (left) and along $z$ axis (right) of a beam with aspect ratio one from solutions of the integrated Green function method, the standard Green function method, and the analytical method.
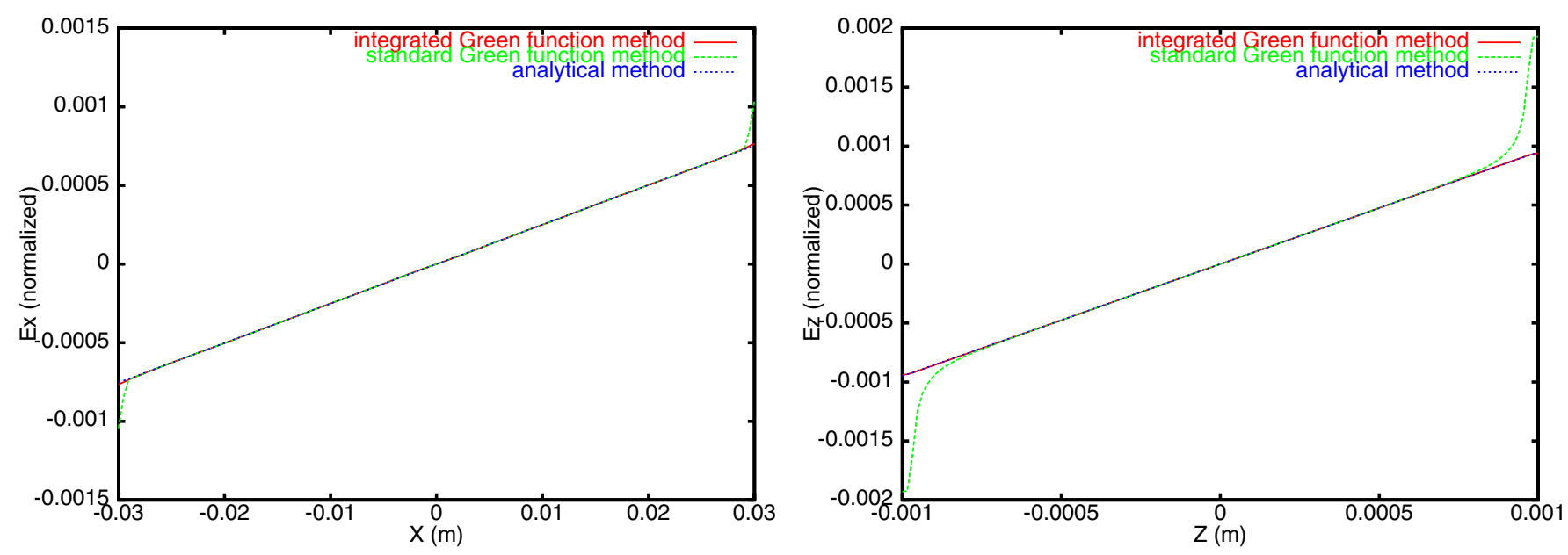

FIG. 5. (Color) Electric fields along $x$ axis (left) and along $z$ axis (right) of an uniform ellipsoidal beam with aspect ratio 30 from solutions of the integrated Green function method, the standard Green function method, and the analytical method.

crepancy for the electric field along $z$ axis, near the edge. For a beam with a Gaussian distribution, the major discrepancy of the electric field occurs around the core, which is given in Fig. 6. These errors in the calculation of electric field for a large aspect ratio beam using the standard Green function method could significantly affect the accuracy of the beam dynamics simulation inside the rf photoinjector. Figure 7 shows a comparison of the transverse and longitudinal rms size of an electron beam in the $S$-band photoinjector using the standard Green function method and the integrated Green function method in the space-charge force calculation using the same number of grid points. The longitudinal rms size from the standard Green function method is significantly larger than that from the integrated Green function method. This is due to an error observed in solving the Poisson equation and calculating the electric field in longitudinal direction using the standard Green function method as shown in Figs. 5 and 6. The calculation

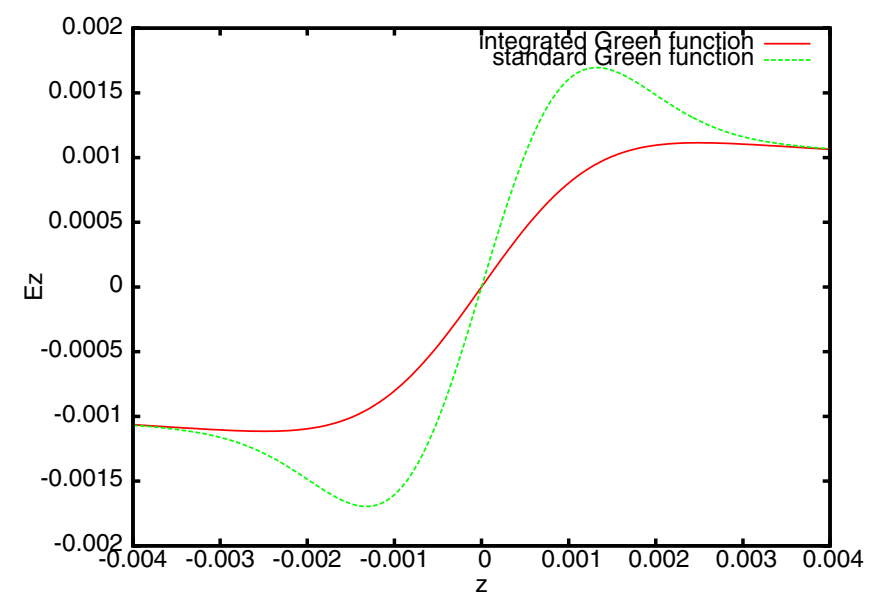

FIG. 6. (Color) Electric fields along $z$ axis of a Gaussian beam with aspect ratio 30 from solutions of the integrated Green function method and the standard Green function method. 

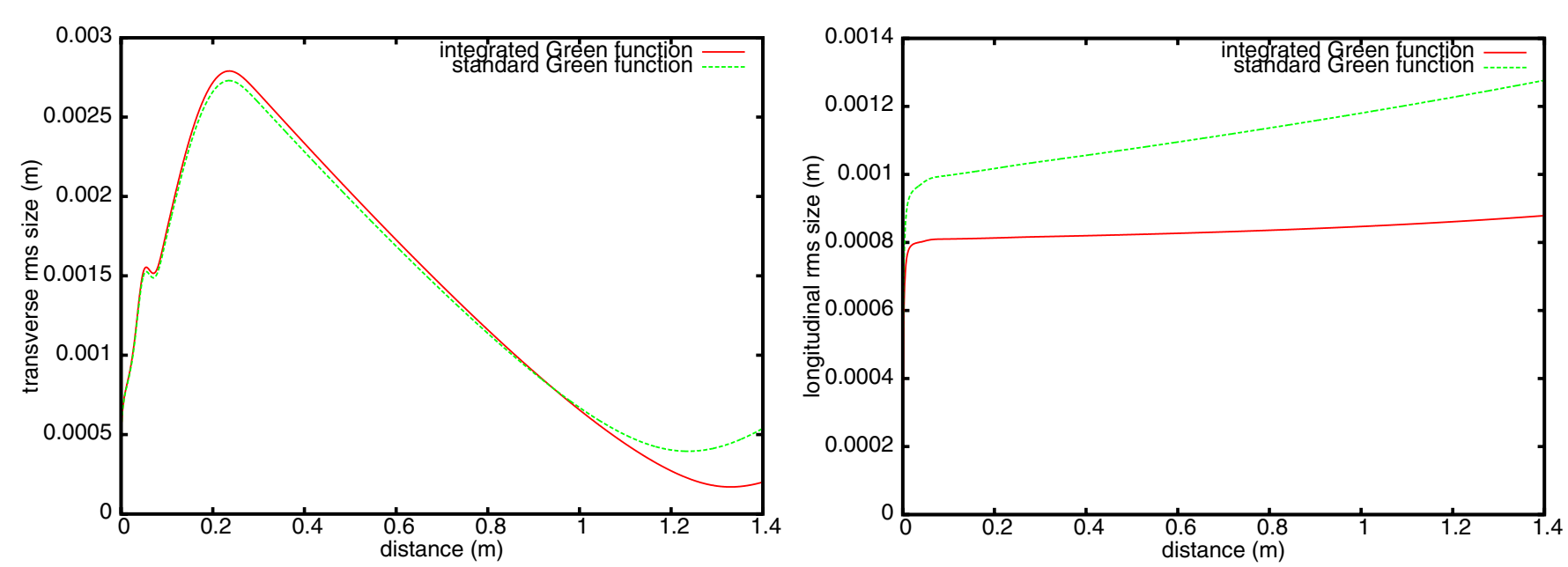

FIG. 7. (Color) Transverse (left) and longitudinal (right) rms sizes as a function of distance from the simulation using the standard and integrated Green function method.

of the integrated Green function in Eq. (16) involves more function operations than that of the standard Green function in Eq. (4). Hence it is also more time consuming than the calculation of the standard Green function. However, this does not affect the $N \log (N)$ scaling law of the original three-dimensional FFT algorithm. Figure 8 shows the computing time for solving the three-dimensional Poisson equation as a function of grid point number using the FFT based integrated Green function method. It is seen that the computing time scales nearly linearly with the number of grid points due to the small $\log (N)$ contribution. Here, $N$ is the number of grid points in one dimension. The integrated Green function method for solving the Poisson equation is slower than the standard Green function method due to the fact that more computational operations

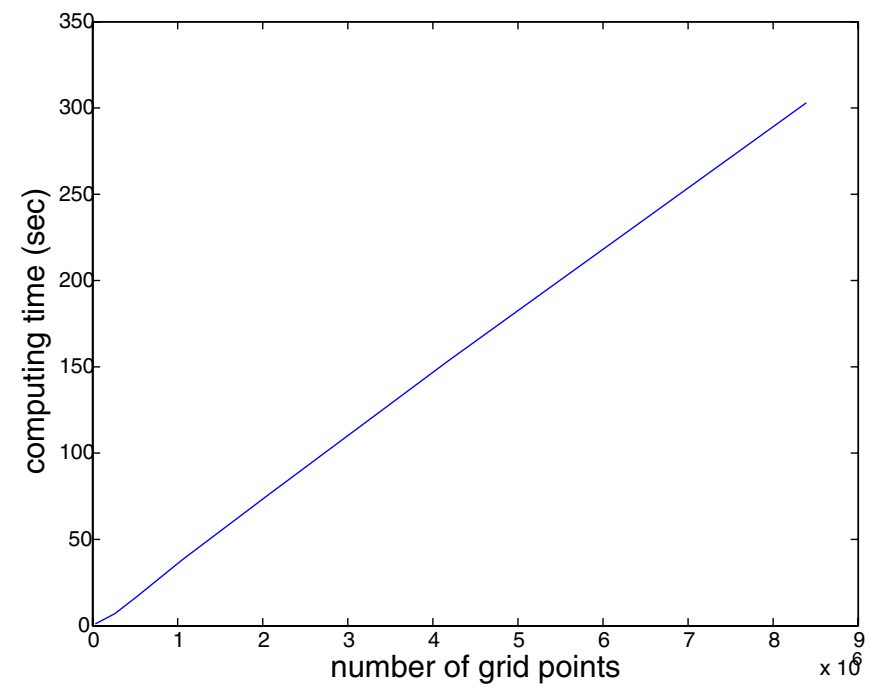

FIG. 8. (Color) Computing time of solving the 3D Poisson equation as a function of number of grid points using the FFT based integrated Green function method. is required to calculate the effective Green function in Eq. (16). However, the cost of computing the Green function will get smaller as more processors are used and the size of domain on each processor gets smaller. For some applications where the size of computational domain is fixed, the Green function is computed once at the beginning. The time difference between the standard Green method and the integrated Green function method will be very small compared with the total computing time.

In photoinjectors, for a finite length input laser pulse, the electron emitted from the photocathode at head of the beam can have significantly different momentum from the electron at the tail if the acceleration gradient is high. In this case, to calculate the space-charge forces by solving the Poisson equation in a single beam frame is not sufficient since there is no single Lorentz transform available in which the spread in longitudinal particle velocities is nonrelativistic [27]. If we divide the initial laser pulse into a number of slices, each slice having a very short pulse length, this results in a small velocity spread within each slice. The Poisson equation can be solved in the beam frame of each slice and the electromagnetic fields are Lorentz transformed back to the laboratory frame for each slice. The total space-charge fields at a given location are then added up from the contribution of each slice. Figure 9 shows the transverse and longitudinal rms sizes of an electron beam with an initial 10 ps flat top laser pulse and $1 \mathrm{nC}$ charge in an $S$-band photoinjector. It is seen that the simulation results converge between two and four slices. The results using only one slice shows about $10 \%-30 \%$ different beam sizes at the exit of the gun. This suggests that for such a beam with $10 \mathrm{ps}$ pulse length, $1 \mathrm{nC}$ electron and $120 \mathrm{MV} / \mathrm{m}$ peak accelerating field, more than two slices (i.e., energy bins) are needed in order to obtain accurate simulation results.

The above three-dimensional quasistatic model has been implemented in a parallel particle-in-cell code, IMPACT-T. 

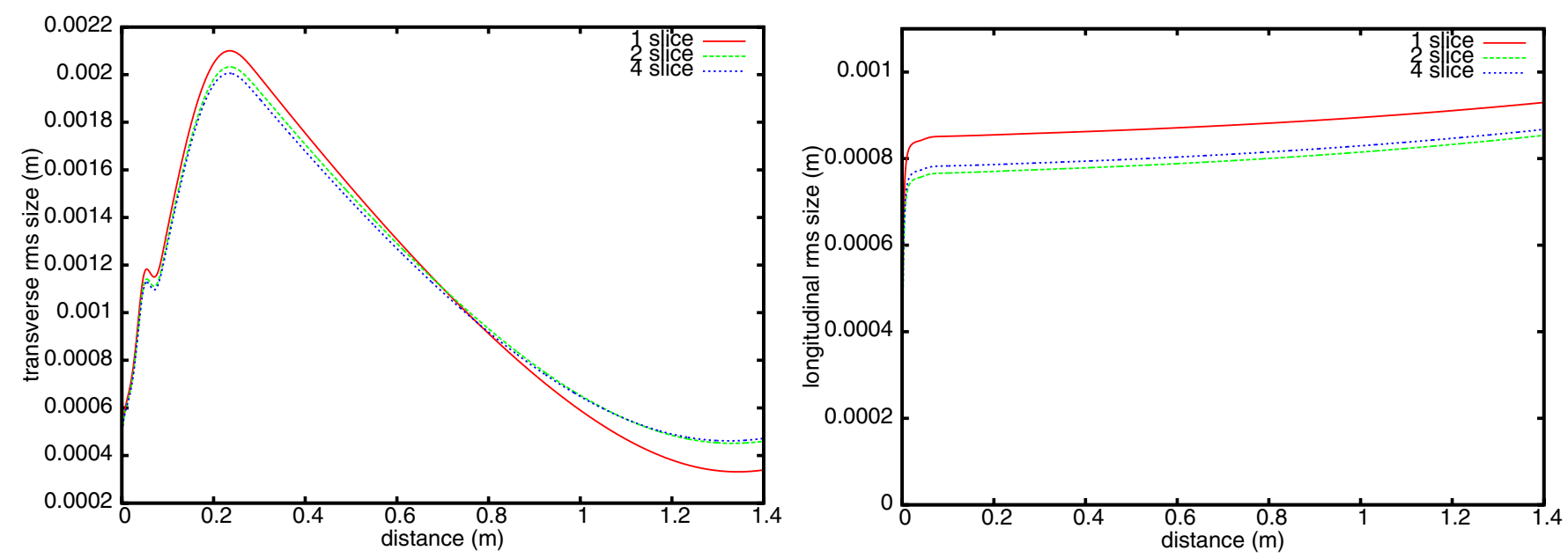

FIG. 9. (Color) Transverse (left) and longitudinal (right) rms sizes as a function of distance using one, two and four slices in the simulation.

The details of parallel implementation of the model is based on a domain-decomposition method which has been discussed in previous publication [28]. The performance of the model on parallel computer is measured and given in Fig. 10. It shows the speedup as a function of number of processors on an IBM-SP3 supercomputer located at the National Energy Research Scientific Computing Center. Here, speedup is defined as a ratio of the computing time on one processor to the computing time on a given number of processors. In the case of perfect scalability, the speedup will be a linear function of the number of processors. It can be seen that the code has a pretty good scalability up to 64 processors. The decreasing of parallel efficiency beyond that number of processor is due to the increasing communication cost associated with the particle movement across processors and the matrix

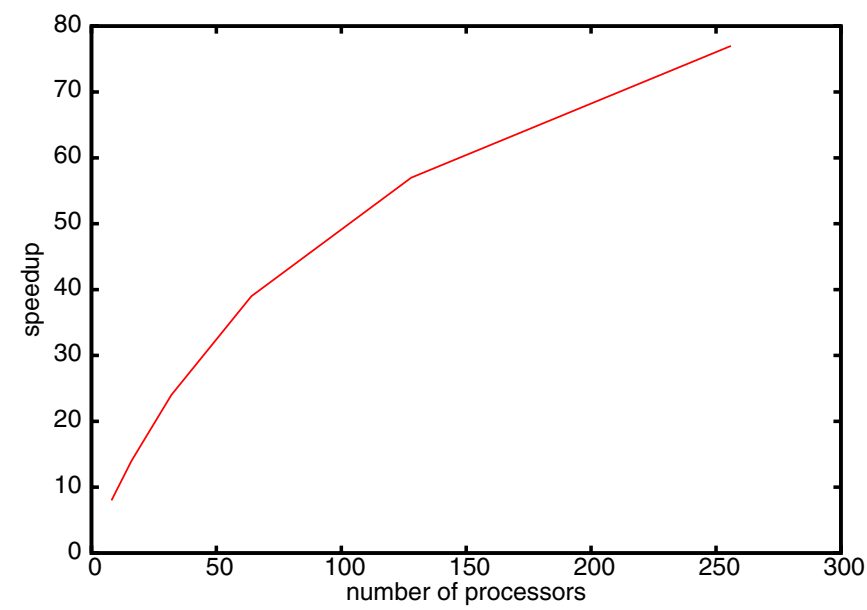

FIG. 10. (Color) Speedup of the parallel implementation as a function of processor number on an IBM SP3 supercomputer. transpose used in the parallel FFT algorithm to solve the Poisson equation.

\section{APPLICATIONS}

As an application, we have studied the effects of initial beam misalignment with respect to $\mathrm{rf}$ accelerating axis on the beam emittance in one LCLS photoinjector design using the three-dimensional model discussed in this paper. The photoinjector in this study consists of a one-and-half cell $2.856 \mathrm{GHz} S$-band gun, an 85 cell traveling wave structure, and two solenoid magnets for initial emittance compensation at low energy and transverse focusing [23]. The peak electric field on the cathode is 120 and $18 \mathrm{MV} / \mathrm{m}$ inside the linac. The electrons out of the photocathode are accelerated to $62 \mathrm{MeV}$ at the end of the traveling wave structure. The initial laser pulse is assumed to have a $10 \mathrm{ps}$ flat top longitudinal density distribution with zero energy spread. The transverse density distribution is assumed to be uniform within a round cross section with $1.2 \mathrm{~mm}$ radius and $0.72 \mathrm{~mm}$ mrad thermal emittance. Figure 11 shows the normalized transverse rms emittances as a function of distance using one slice, two slice, four slice, and eight slice model of the beam. The simulations were done using about $10^{6}$ macroparticles with $64 \times 64 \times 64$ mesh points. We have varied the number of macroparticles, the mesh points and the time step size to ensure the convergence of simulation results. It appears that four slices are needed to model the beam for numerical convergence. The final emittance at the end of $5.0 \mathrm{~m}$ is about $1.4 \mathrm{~mm} \mathrm{mrad}$ from the four slice model, which is larger than the $1.1 \mathrm{~mm}$ mrad from the single slice model. This emittance could be further reduced with some fine tuning of lattice parameters such as magnetic focusing strength. The simulation took about $4.5 \mathrm{~h}$ computing time on 64 IBM-SP3 processors with $375 \mathrm{MHz}$ clock speed without special machine opti- 


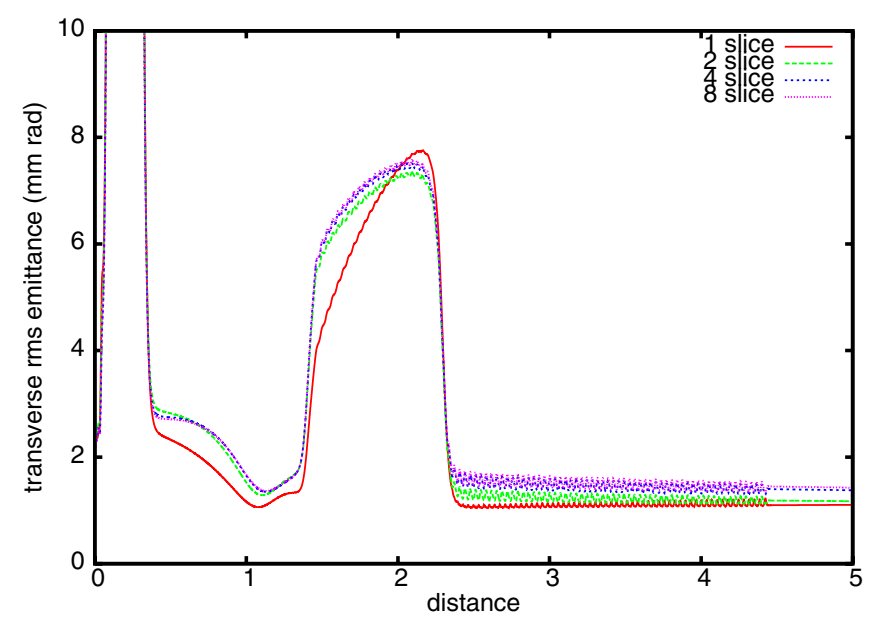

FIG. 11. (Color) Transverse normalized rms emittance as a function of distance using one slice, two slice, four slice, and eight slice model of the beam.

mization. The rms emittance is calculated using the mechanical momenta of individual particles in the laboratory frame.

In real photoinjector operation, the center of laser spot on the photocathode may not be exactly coincident with the rf structure axis. This causes initial misalignment of photo electron beam inside the rf gun. Figure 12 shows the emittance growth at the end of the injector as a function of initial misalignment of the beam. It can be seen that the averaged transverse emittance grows quickly with the increase of the initial misalignment errors. To keep the averaged emittance growth below the $10 \%$ level, the initial misalignment has to be controlled within $0.2 \sigma$. In these simulations, the centroid offset was corrected by assumed steering magnets so that at the entrance to traveling wave linac structure, the bunch centroid is set back to zero

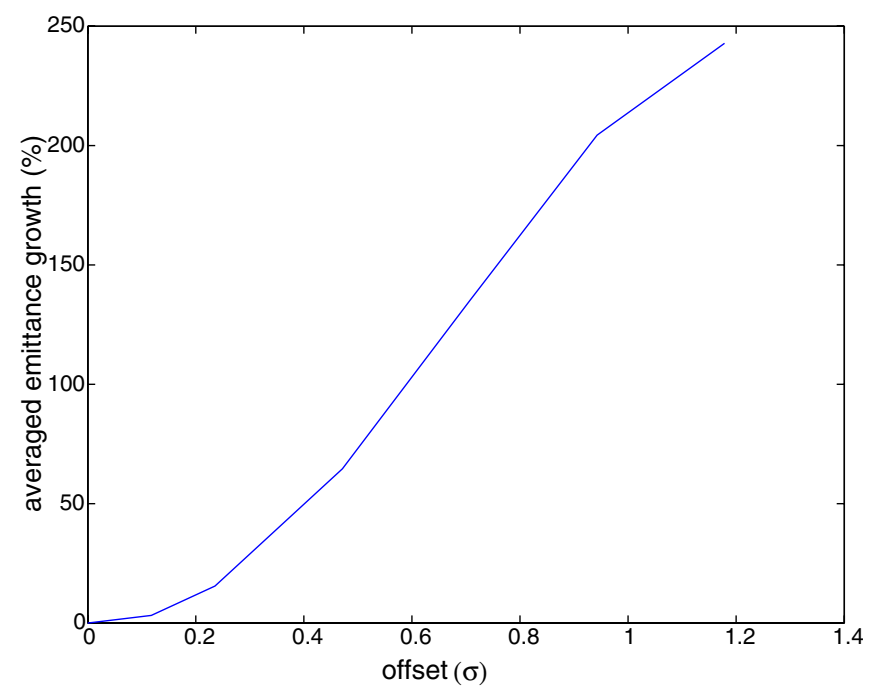

FIG. 12. (Color) Emittance growth at the end of the injector as a function of initial misalignment of the beam. numerically. The effects of wakefields are not included in these simulations.

\section{CONCLUSIONS}

In this paper, we have presented a three-dimensional quasistatic model for high brightness beam dynamics simulation on parallel computers. It is unique in its use of space-charge solvers based on an integrated Green function to efficiently and accurately treat threedimensional beams with large aspect ratio, and a shiftedGreen function to efficiently treat image-charge effects of a cathode. We have shown that the standard Green function method is not accurate enough to handle the high brightness beam especially during the emission when the transverse-to-longitudinal aspect ratio is large. We have also shown that the image space-charge effects from conducting photocathode are important to the beam quality inside photoinjector and can be efficiently treated using a shifted-Green function method. The model presented in this paper is also unique in its inclusion of energy binning in the space-charge calculation to model beams with large energy spread. We have shown that more than two energy slices are needed to accurately simulate a $1 \mathrm{nC}$ beam with $10 \mathrm{ps}$ initial length and going through $120 \mathrm{MV} / \mathrm{m}$ rf accelerating gradient. The above numerical algorithms are implemented in a parallel particle-in-cell code. More than $50 \%$ parallel efficiency has been achieved up to 64 processors on an IBM-SP3 computer. Together, all these features make it a powerful and versatile tool for modeling threedimensional beams from inhomogeneous emission, beams subject to initial misalignment and applications related to next generation linear colliders. As an application, we have studied the beam transport in one LCLS photoinjector design through the first traveling wave linac with initial misalignment errors. It suggests that the misalignment error should be controlled within $0.2 \sigma$ to keep the averaged emittance growth below $10 \%$ even without including the effects of wakefields.

\section{ACKNOWLEDGMENTS}

We would like to thank Dr. M. A. Furman for reading the manuscript. This research used resources of the National Energy Research Scientific Computing Center, and the resources of the Center for Computational Sciences at Oak Ridge National Laboratory. This work was supported by the U.S. Department of Energy under Contract No. DEAC02-05CH11231, and by a Scientific Discovery through Advanced Computing project, "Advanced Computing for 21 st Century Accelerator Science and Technology," which is supported by the U.S. DOE/SC Office of High Energy Physics and the Office of Advanced Scientific Computing Research. 
[1] K. J. Kim, Nucl. Instrum. Methods Phys. Res., Sect. A 275, 201 (1989).

[2] J. Gao, Nucl. Instrum. Methods Phys. Res., Sect. A 297, 335 (1990).

[3] L. Serafini and J. B. Rosenzweig, Phys. Rev. E 55, 7565 (1997).

[4] L. Young and J. Billen, in Proceedings of the Particle Accelerator Conference, Portland, OR, 2003 (IEEE, Piscataway, NJ, 2003), p. 3521.

[5] K. Flottmann and P. Piot, in Proceedings of the 8th European Particle Accelerator Conference, Paris, 2002 (EPS-IGA and CERN, Geneva, 2002), pp. 1798-1800.

[6] S. B. Van der Geer and M. J. de Loos, in Proceedings of the 1997 Particle Accelerator Conference, Vancouver, Canada (IEEE, Piscataway, NJ, 1998), p. 2577.

[7] L. Giannessi et al., in Towards X-Ray Free Electron Lasers: Workshop on Single Pass, High Gain FELs Starting from Noise, Aiming at Coherent X-Rays, edited by R. Bonifacio and W. A. Barletta, AIP Conf. Proc. No. 413 (AIP, New York, 1997), p. 301.

[8] J. L. Coacolo et al., Nucl. Instrum. Methods Phys. Res., Sect. A 393, 430 (1997).

[9] L. Serafini and C. Pagani, in Proceedings of the 1st European Particle Accelerator Conference, Rome, Italy, 1988 (EPS-AG and CERN, Geneva, 1988), p. 866.

[10] B. Goplen et al., Comput. Phys. Commun. 87, 54 (1995).

[11] Y. K. Batygin, in Proceedings of the 3rd European Particle Accelerator Conference, Berlin, Germany, 1992 (EPS-AG and CERN, Geneva, 1992), p. 822.

[12] C. Limborg et al., in Proceedings of the Particle Accelerator Conference, Portland, OR, 2003 (IEEE, Piscataway, NJ, 2003), p. 3548.

[13] D. J. Gibson, F. V. Hartemann, E. C. Landahl, A. L. Troha, N. C. Lumann, G. P. Le Sage, and C. H. Ho, Phys. Rev. ST Accel. Beams 4, 090101 (2001).
[14] Y.E. Sun, P. Piot, K. J. Kim, N. Barov, S. Lidia, J. Santucci, R. Tikhoplav, and J. Wennerberg, Phys. Rev. ST Accel. Beams 7, 123501 (2004).

[15] M. Krasilnikov et al., in Proceedings of the European Particle Accelerator Conference, Lucerne, Switzerland, 2004 (EPS-AG and CERN, Geneva, 2004), p. 360.

[16] J. Qiang, M. A. Furman, and R. D. Ryne, J. Comput. Phys. 198, 278 (2004).

[17] L. Serafini, in The Physics of High Brightness Beams, edited by J. Rosenzweig and L. Serafini (World Scientific, Singapore, 2000), p. 27.

[18] E. Colby, V. Ivanov, Z. Li, and C. Limborg, in Proceedings of ICAP 2002: The 7th International Computational Accelerator Physics Conference, East Lansing, Michigan (Institute of Physics, London, 2002), p. 47.

[19] G. A. Loew, R.H. Miller, R. A. Early, and K. L. Bane, Report No. SLAC-PUB-2295, 1979.

[20] P.L. Morton, Ph.D. thesis, The Ohio State University, 1963.

[21] S. M. Lidia, Lawrence Berkeley National Laboratory Report No. LBNL-45254, 1999.

[22] R. W. Hockney and J. W. Eastwood, Computer Simulation Using Particles (Adam Hilger, New York, 1988).

[23] C. Limborg-Deprey, S. Gierman, and D. Dowell, in Proceedings of the European Particle Accelerator Conference, Lucerne, Switzerland, 2004 (Ref. [15]), p. 521.

[24] K. Ohmi, Phys. Rev. E 62, 7287 (2000).

[25] V. Ivanov (private communication).

[26] R.D. Ryne, contribution to the ICFA Beam Dynamics Mini Workshop on Space Charge Simulation, Trinity College, Oxford, 2003 (unpublished).

[27] G. Fubiani et al., in Proceedings of Advanced Accelerator Concepts, 2002 (AIP, Melville, NY, 2002), p. 203.

[28] J. Qiang, R. D. Ryne, S. Habib, and V. Decyk, J. Comput. Phys. 163, 434 (2000). 